\title{
Diferentes proposições do princípio da incerteza para posição e momentum: integrando formalismo matemático, fenomenologia e interpretações no ensino da teoria quântica
}

Different propositions of the uncertainty principle for position and momentum: integrating mathematical formalism, phenomenology and interpretations in the teaching of quantum theory

\author{
Gabriela Gomes Rosa ${ }^{* 10}$, Nathan Willig Lima ${ }^{1 @}$, Cláudio José de Holanda Cavalcanti1@ \\ ${ }^{1}$ Universidade Federal do Rio Grande do Sul, Instituto de Física, Porto Alegre, RS, Brasil.
}

Recebido em 19 de agosto de 2021. Revisado em 22 de novembro de 2021. Aceito em 04 de dezembro de 2021.

\begin{abstract}
O Princípio da Incerteza, proposto pelo físico Werner Heisenberg em 1927, possui centralidade no desenvolvimento Teoria Quântica e desde sua formulação assume uma pluralidade de interpretações, derivações matemáticas e experimentos mentais que buscam explorá-lo em profundidade. Entretanto, os livros didáticos, que possuem papel importante na formação de cientistas e professores de ciência, usualmente optam por privilegiar somente alguns de seus principais aspectos, limitando a apresentação do Princípio da Incerteza nos cursos de Teoria Quântica do ensino superior. Com o objetivo de ampliar as discussões sobre o Princípio da Incerteza para as variáveis posição e momentum e contribuir com o Ensino da Teoria Quântica, ao longo deste texto apresentamos o Princípio da Incerteza em três diferentes níveis (formalismo matemático, fenomenologia e interpretação). Começamos resgatando de forma didática quatro diferentes derivações históricas do Princípio da Incerteza: as derivações de Heisenberg e Weyl para as variáveis posição e momentum, e as derivações de Robertson e Schrödinger para dois observáveis quaisquer A e B. Em seguida, retomamos dois experimentos mentais propostos por Heisenberg: o microscópio de raios-gama e o elétron passando por uma fenda. Por fim, apresentamos quatro principais escolas de interpretação do Princípio da Incerteza.
\end{abstract}

Palavras-chave: Princípio da Incerteza, Teoria Quântica, Ensino de Física.

The Uncertainty Principle, proposed by the physicist Werner Heisenberg in 1927, is central to the development of Quantum Theory and since its summary assumes a plurality of interpretations, mathematical derivations and mental experiments that seek to explore it in depth. However, textbooks, which play an important role in the training of scientists and science teachers, usually choose to privilege only some of their main aspects and end up limiting the presentation of the Uncertainty Principle in Quantum Theory courses in higher education. In order to increase discussions about the Uncertainty Principle for the variables position and moment and contribute to the Quantum Theory Teaching, throughout this text we present the Uncertainty Principle at three different levels (mathematical formalism, phenomenology and interpretation). We started by rescuing, in a didactic way, four different historical derivations of the Uncertainty Principle: the Heisenberg and Weyl derivations for the variables position and momentum, and the Robertson and Schrodinger derivations for two observables any A and B. Then, we retake two thought experiments proposed by Heisenberg: the gamma-ray microscope and the electron passing through a slit. Finally, we present the four main schools of interpretation of the Uncertainty Principle.

Keywords: Uncertainty Principle, Quantum Theory, Physics Teaching.

\section{Introdução}

O Princípio da Incerteza foi, primeiramente, formulado por Werner Heisenberg [1] em 19271 No ano seguinte, Niels Bohr [2] incorporou o Princípio da Incerteza como um dos elementos principais do que ficou conhecimento

\footnotetext{
*Endereço de correspondência: gabrielagomesr@outlook.com

1 O termo "Princípio da Incerteza" só foi proposto ao final de 1927 por A. E. Ruark [2] e, embora seja amplamente utilizado, não condiz com o fenômeno em si. O que conhecemos e ensinamos como Princípio da Incerteza não se trata de um princípio de fato, mas de uma relação de incerteza que se estende a qualquer sistema descrito por ondas e, dessa forma, se aplica também a outras variáveis.
}

como Interpretação da Complementaridade - tornandose um elemento fundamental da Teoria Quântica. Apenas para fornecer alguns exemplos da centralidade do Princípio da Incerteza na Teoria Quântica, em 1933, em seu famoso livro didático sobre Mecânica Quântica, Pauli [3] a introduz por meio do Princípio da Incerteza e da Complementaridade. Em 1935, no artigo de Einstein, Podolski e Rosen [4, que ficou conhecido como paradoxo EPR, os autores apresentam a interpretação usual da Teoria Quântica ressaltando o fato de que variáveis que não comutam não podem ser conhecidas simultaneamente com qualquer precisão. Em 1952, no seu artigo sobre variáveis ocultas, David Bohm menciona 
"A interpretação física usual da teoria quântica se centra em torno do Princípio da Incerteza" (1952, p. 167, tradução livre) [5]. Apesar de tal centralidade, os livros didáticos contemporâneos de Mecânica Quântica, muitas vezes, tratam o tema de forma periférica ou, pelo menos, não discutem em detalhes as diferentes formas de se derivar as relações de incerteza, os diferentes experimentos mentais que podem ser mobilizados para entendê-lo e, sobretudo, as diferentes interpretações que ele enseja. Ademais, em um estudo preliminar [6], mostramos que os livros didáticos usualmente fazem uma escolha: (1) privilegiam a apresentação do formalismo matemático em profundidade ou (2) enfatizam os aspectos históricos e interpretativos da teoria.

Embora exista uma ampla literatura voltada para discussões acerca do Princípio da Incerteza, incluindo diferentes derivações e generalizações do princípio [7] [1], estudos voltados para discussões fenomenológicas, centrados no experimento mental do microscópio de raios-gama proposto por Heisenberg em 1927 [18 21] e, ainda, trabalhos que buscam discutir conceitualmente o Princípio da Incerteza, suas implicações teóricas e possíveis interpretações, 2225], a maioria dos trabalhos costuma ser voltada a pesquisadores e estudiosos da área, tornando pouco provável sua utilização como instrumento auxiliar para o ensino da Teoria Quântica. Na literatura nacional já existem estudos voltados para a discussão de aspectos matemáticos [26] e filosóficointerpretativos do Princípio da Incerteza [27.

Neste contexto, com o presente trabalho, buscamos contribuir para o ensino desse tópico central da Teoria Quântica ao fornecer um panorama geral e uma proposta de exploração didática. Reconhecendo a dificuldade dos alunos em compreender o formalismo da Teoria Quântica [28], apresentamos aqui um texto de caráter didático que, ao abordar diferentes aspectos do Princípio da Incerteza, é organizado em três categorias: formalismo matemático, fenomenologia e interpretação, com o intuito de que tal abordagem favoreça sua aprendizagem. Em especial, apresentamos diferentes derivações inspiradas em textos históricos do Princípio da Incerteza (explicando passos que foram omitidos nos textos originais e organizando a apresentação de forma detalhada), retomamos a explicação de dois experimentos mentais imaginados por Heisenberg para explicar o princípio da incerteza 1] e apresentamos quatro principais escolas de interpretação do princípio [23].

Essa apresentação é feita a partir dos próprios trabalhos originais da Teoria Quântica (fontes históricas primárias) e de comentários já existentes (fontes históricas secundárias). Tal texto pode ser introduzido em disciplinas de Mecânica Quântica, após a apresentação tradicional, contribuindo para complementar o que foi estudado no livro didático e para enriquecer as discussões e compreensões sobre tal princípio. Essa proposta, nesse sentido, se alinha aos trabalhos que propõem trazer fontes primárias para o Ensino de Física como uma forma de enriquecer a atividade didática e adotar um pluralismo metodológico e didático [29] 32 . A escolha de apresentar o texto didático dividido em três categorias (formalismo matemático, fenomenologia, e interpretação) é inspirada na discussão feita por Max Jammer 33 sobre os elementos de uma teoria física e dialoga com trabalhos da área de Ensino de Física.

O texto está organizado da seguinte forma: na seção2 apresentamos uma breve discussão sobre a proposta didática de discutir conceitos físicos em três categoria, explicamos cada uma das categorias e justificamos a importância de apresentá-las de forma clara no contexto pedagógico. Na seção 3. fazemos um breve resgate histórico da gênese do Princípio da Incerteza, para situar o leitor no momento histórico do desenvolvimento das discussões que aparecem na sequência. Na seção 4 , introduzimos o primeiro nível, o formalismo matemático, resgatando de forma didática quatro derivações históricas do Princípio da Incerteza. Na sequência, na seção 5. explicamos dois experimentos mentais mencionados no artigo de 1927 de Heisenberg, explicando detalhes que não aparecem no artigo original. Na seção 6, apresentamos quatro formas distintas de se interpretar o princípio da incerteza. Por fim, na seção 7] apresentamos nossas considerações finais, fazendo algumas sugestões para o uso didático desse texto.

\section{Proposta Didática: Apresentando Conceitos Físicos em Três Categorias (Formalismo Matemático, Fenomenologia e Interpretações)}

A proposta didática de discutir conceitos físicos em três categorias é inspirada na discussão levantada por Max Jammer 33 no livro "The Philosophy of Quantum Physics" em que o autor propõe que teorias físicas são constituídas por, pelo menos, três componentes distintos: formalismo abstrato, regras de correspondência e interpretações. O formalismo abstrato é essencialmente a estrutura lógica de uma teoria, seus postulados desprovidos de significado empírico. Por meio das regras de correspondência, o formalismo abstrato deixa de ser um conjunto de símbolos e passa a representar um sistema físico. Na teoria quântica, por exemplo, o que usualmente é chamada de interpretação probabilística de Born pode ser considerada como a regra de correspondência, pois conecta um elemento abstrato da teoria, a função de onda, com uma grandeza mensurável, a probabilidade de se encontrar uma partícula em uma certa região. Por fim, Jammer [33] aponta que as teorias físicas ainda possuem um terceiro elemento, sua interpretação, a qual contempla o conjunto de enunciados da teoria que descrevem a realidade (como modelos utilizados) mas que não alteram as previsões experimentais da teoria 34 .

Dessa forma, propomos que tal estrutura não se restrinja ao estudo de teorias físicas, mas que possa 
ser ampliada para o estudo de conceitos e princípios físicos em um contexto mais amplo, tal como o Princípio da Incerteza, e de maneira análoga a Max Jammer, sugerimos que sejam apresentados em três diferentes categorias: formalismo matemático, fenomenologia e interpretações ${ }^{2}$

Os conceitos apresentados na Teoria Quântica não são simples e, muitas vezes, contrariam nossas intuições mais fundamentais, de modo que os estudantes em geral costumam ter dificuldade para compreender sua estrutura [28]. Por esse motivo, apresentar tais conceitos em três categorias pode contribuir para uma melhor organização da apresentação didática ${ }^{3}$ Utilizando dessa apresentação, primeiramente, o aluno entende como o Princípio se desenvolve a partir do formalismo matemático, aprendendo como que ele se conecta com a estrutura geral da Teoria. Na sequência, são discutidos aspectos fenomenológicos, ou seja, resultados empíricos obtidos em experimentos com sistemas quânticos. Nesse momento não é discutido o que o princípio significa, mas como que os resultados experimentais traduzem tal princípio. Somente então, nas situações em que o professor achar necessário e tiver interesse, discutese a interpretação desse formalismo e seus resultados. Ressaltamos que a abordagem do terceiro nível deve ser considerada como uma escolha para professores e professoras que considerem as discussões filosóficas como frutíferas ao Ensino de Física e que encontrem espaço para elas em suas aulas.

\section{Um Breve Histórico da Gênese do Princípio da Incerteza}

No início do século XX, os estudos sobre matéria e radiação conduziram a uma série de resultados que contrariavam concepções fundamentais da Física Clássica. Em especial, a radiação, usualmente tratada por campos contínuos, passou também a ser representada por uma caracterização corpuscular, como nos trabalhos de Einstein, e a matéria, usualmente tratada de forma corpuscular também ganhou um caráter ondulatório, como nos trabalhos de Louis de Broglie. Essas representações contraintuitivas, embora fossem capazes de explicar uma série de resultados experimentais, não conseguiam formar um quadro teórico completo e consistente. $\mathrm{Na}$ metade da segunda década do século XX, dois programas de pesquisa surgiram justamente com o objetivo de articular tal quadro completo e coerente [2].

Um desses programas foi inaugurado em 1925 pelo físico alemão Werner Heisenberg por meio do desenvolvimento da Mecânica Matricial que, inspirada por ideias

\footnotetext{
2 Proposta semelhante, mas subsidiada por diferentes razões teóricas, foi apresentada para a dualidade onda-partícula para dialogando com o que já foi proposto para dualidade ondapartícula por Cheong \& Song [35].

3 Embora tal separação seja artificial e não reflita toda a complexidade envolvida na estabilização de um conceito ou uma teoria física.
}

consistentes com a teoria da relatividade de Einstein [2], com sua essência centrada na concepção corpuscular da matéria e na descontinuidade dos processos atômicos, propôs um formalismo completo e totalmente novo para a física quântica. Em seu texto, Heisenberg rejeitou as noções clássicas de posição e velocidade (ou momentum) para elétrons em átomos, trabalhando apenas com grandezas observáveis, e empregou o princípio da correspondência de Bohr no cerne de seu esquema matemático [36].

Apesar do sucesso de Heisenberg em descrever o comportamento dos fenômenos quânticos utilizando da Mecânica Matricial, a comunidade científica, em geral, relutou em aderir a sua formulação, tanto pela estranheza ao formalismo matemático quanto pela abstração teórica do artigo de 1925 [2, 37]. Entretanto, tal abstração chamou a atenção de alguns físicos teóricos da época, resultando em um maior desenvolvimento da Mecânica Matricial pelos físicos Born e Jordan [38, 39], Pauli [40] e Dirac 41] de maneira que, finalmente, foi formulada a teoria de Dirac-Jordan ${ }^{4} 42,45$.

No ano seguinte, partindo do teorema de Broglie sobre a equivalência entre o Princípio de Maupertuis e o Princípio de Fermat, o físico austríaco Erwin Schrödinger desenvolveu a Mecânica Ondulatória em uma série de quatro artigos [46] provando, nos três primeiros, que a Mecânica Ondulatória era matematicamente equivalente à Mecânica Matricial. Entretanto, apesar da equivalência formal entre os dois programas, a interpretação da teoria e a maneira como cada uma explicava os fenômenos quânticos levantou um dilema irreconciliável, uma vez que Heisenberg e Schrödinger utilizavam de diferentes estruturas para descrever a realidade, reforçando os paradoxos entre a representação corpuscular e ondulatória da mecânica quântica. Enquanto Heisenberg e a Mecânica Matricial representavam os objetos quânticos por meio de fenômenos corpusculares, Schrödinger e a Mecânica Ondulatória centravam-se na ideia metafísica de que qualquer partícula é representada por um conjunto de ondas, de modo que a descrição da dinâmica da partícula seria feita por meio da descrição do transporte dessas ondas.

Principalmente por apresentar um formalismo matemático com o qual os físicos já estavam adaptados [2, 37], baseado na resolução de equações diferenciais parciais e compatível com a Teoria de Hamilton-Jacobi, a formulação matemática da Mecânica Ondulatória de Schrödinger rapidamente recebeu a aprovação da comunidade científica [2, 33], sendo adotada como formalismo hegemônico da teoria quântica, presente majoritariamente, até hoje, nos livros didáticos e nos cursos de formação em física quântica.

\footnotetext{
${ }^{4}$ Embora não esteja presente no estudo contemporâneo da Teoria Quântica, e sua contribuição dificilmente será encontrada em algum livro didático, destacamos aqui sua importância pois, segundo o próprio Heisenberg no artigo de 1927, o Princípio da Incerteza foi primeiramente formulado utilizando da teoria de Dirac-Jordan [1].
} 
Por outro lado, a interpretação física de tal formalismo não foi tão bem aceita, principalmente no que se refere a sua interpretação para a função de onda, que trazia uma série de problemas [2], sendo a principal contradição encontrada no pacote localizado, uma vez que a redução do pacote de onda por si só já estaria associada a um efeito não-local 34 .

Nesse período, ademais, a natureza estatística das transições atômicas passou a chamar a atenção dos físicos para a necessidade de um rompimento com características importantes da Física Clássica. Em 1925, por exemplo, Einstein [47] deu a primeira interpretação estatística às ondas de de Broglie, uma vez que, ao tentar tornar a dualidade onda-partícula mais compreensível, interpretou o quadrado das amplitudes das ondas ópticas como densidade de probabilidade para detectar um fóton em uma dada região [37. A descrição probabilística da realidade foi posteriormente desenvolvida nas obras de Born, Pauli e Jordan no período de 1925 a 1927. Apropriando-se do formalismo ondulatório de Schrödinger e associando-o a uma interpretação corpuscular da matéria, e tendo como base o estudo dos processos de colisões atômicas, Max Born propôs que a função de onda $\Psi$ deveria representar a densidade de probabilidade para elétrons (ou outras partículas) ${ }^{5}$ [37, de modo que a probabilidade de detectar um único quantum em determinada região do espaço seria proporcional ao quadrado da amplitude $\Psi$ da onda associada à região.

Conforme Heisenberg [49, foi no início de 1926 que ele tomou conhecimento das ideias de Schrödinger e, ao final do período letivo o mesmo ano, teve a oportunidade de assistir uma palestra do físico austríaco em Munique. Em seu livro 49, o físico alemão aponta que ficou inconformado com o fato de a Mecânica Ondulatória negar a descontinuidade dos processos atômicos, e que, após a palestra, levantou uma série de objeções à teoria. Entretanto, conforme Heisenberg [49] destaca, suas contestações foram isoladas e, de modo geral, a Mecânica Ondulatória foi bem aceita pelo público de físicos presentes. Em setembro do mesmo ano Niels Bohr convidou Schrödinger para visitar Copenhague com o objetivo de discutir, em detalhes, as duas diferentes interpretações. Segundo Heisenberg [49, 50, foram dias de intermináveis discussões, começando nas primeiras horas da manhã e estendendo-se até tarde da noite. Tais discussões não ajudaram a resolver o impasse entre as duas teorias concorrentes, e cada lado saiu convencido de estar trilhando pelo caminho certo [49, [50]. Nos meses subsequentes, o foco de Bohr e Heisenberg foi dirigido para a busca por uma interpretação física da Mecânica Quântica por meio da discussão de diversos experimentos mentais [50].

Ainda segundo Heisenberg 49], em fevereiro de 1927, enquanto Bohr passava por um período de férias

\footnotetext{
5 Embora em 1954 Max Born alegue que tenha se inspirado na proposta de Einstein para a probabilidade de ocorrência do fóton, Mara Beller 48 traz argumentos que contradizem tal afirmação.
}

esquiando na Noruega, ao tentar descrever matematicamente a trajetória percorrida pelo elétron no experimento da câmara de nuvem, o físico lembrou de uma discussão que havia tido em Gottigen sobre a possibilidade de determinar a posição de uma partícula com um microscópio de raios-gama. Nesse momento, o físico alemão se questionou se a Teoria Quântica poderia representar, com alguma imprecisão, o fato de um elétron se encontrar em determinada posição e se mover com determinada velocidade e, ainda, se existiria a possibilidade de tornar tais imprecisões tão pequenas de modo que não produzissem obstáculos experimentais. Dessa forma, com o objetivo de legitimar a Mecânica Matricial, os saltos quânticos e sua interpretação corpuscular da matéria, Heisenberg publicou o artigo intitulado "The Physical Content of Quantum Kinematics and Mechanics" que, segundo ele, levaria a uma interpretação da mecânica quântica livre de inconsistências e onde definiu, pela primeira vez, o Princípio da Incerteza: "quantidades canonicamente conjugadas podem ser determinadas simultaneamente apenas com uma indeterminação característica" (1983, p. 1, tradução livre) [1].

Sendo fortemente influenciado pela Teoria da Relatividade Especial, Heisenberg propôs que para especificar o que deve ser entendido por "posição de uma partícula" seria necessário determinar o aparato experimental que permite a medida da sua posição, caso contrário este termo não possui significadd ${ }^{6}$ Usando tal metáfora, Heisenberg apresentou alguns experimentos mentais que, de acordo com ele, revelariam a impossibilidade de dois parâmetros conjugados serem medidos simultaneamente com precisão arbitrária e uma demonstração matemática que, conforme ele aponta, foi baseada na teoria de DiracJordan.

\section{Primeiro Nível: O Formalismo Matemático Associado ao Princípio da Incerteza}

Ao longo dessa seção, apresentaremos uma reconstrução didática de quatro derivações inspiradas em textos históricos do Princípio da Incerteza para posição e momentum. Para acompanhar algumas das premissas aqui utilizadas, é recomendável que o leitor já tenha conhecimento prévio dos Postulados da Teoria Quântica, que podem ser retomados no Material Suplementar Apêndice A. Também será necessária certa familiaridade com conceitos da Teoria Estatística, que podem ser consultados no Material Suplementar - Apêndice B.

\footnotetext{
6 Tal como na Relatividade que, ao falar de eventos simultâneos, é necessário que se especifique quais relógios serão utilizados para a realização das medidas de tempo.
} 


\subsection{Incerteza entre as variáveis posição e momentum para um pacote de ondas gaussiano - reconstrução didática da derivação presente no trabalho de Heisenberg (1927)}

Para formalizar matematicamente a relação de incerteza para posição e momentum, no texto de 1927 Werner Heisenberg [1] aponta que utilizou a teoria da Transformação Matricial de Dirac-Jordan (designação do próprio autor), assumindo seu formalismo matemático, mas discordando de sua interpretação original 7 Heisenberg propôs que, ao representar uma partícula utilizando o formalismo de Jordan, com uma função gaussiana, por exemplo, o desvio padrão deveria ser pragmaticamente interpretado como o desvio padrão de uma medição.

Ao longo dessa apresentação utilizaremos um raciocínio similar, mas com uma abordagem contemporânea [51]. Desta forma, temos que, uma partícula quântica pode ser descrita por meio de uma função de onda $\Psi(x, t)$ (Postulado 1 do apêndice $\mathrm{A}$ ), cuja frequência angular e o número de onda podem ser descritos pelas expressões de Einstein e de Broglie

$$
p=\hbar k
$$

onde $k=2 \pi / \lambda$ é o número de onda. E, para a energia,

$$
E=\hbar \omega
$$

No caso de uma partícula livre, toda a sua energia estará associada a seu movimento, o que significa que será puramente cinética

$$
E=\frac{p^{2}}{2 m}
$$

Portanto,

$$
\hbar \omega=\frac{\hbar^{2} k^{2}}{2 m} .
$$

Dessa forma, podemos começar expressando uma função de onda qualquer como uma "soma" de ondas planas. Uma vez que $k$ apresenta um espectro contínuo, podemos expressar a função de onda como uma integral em relação a $k$

$$
\Psi(x, t)=\frac{1}{2 \pi} \int_{-\infty}^{+\infty} g(k) e^{i(k x-\omega t)} d k .
$$

Em que o termo $\frac{1}{2 \pi}$ será explicado mais adiante. Nesse caso, a função $g(k)$ representa o coeficiente de expansão da função de onda em relação aos autoestados.

Ademais, Heisenberg propõe preparar um arranjo experimental em que, em um específico instante, pode-se dizer $t=0$, a partícula estará em torno de uma posição

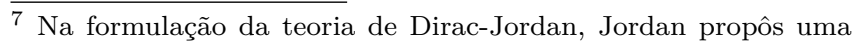
interpretação probabilística para a mesma.
}

média $\langle x\rangle$ com precisão $\sigma / \sqrt{2}$. Podemos traduzir tal arranjo experimental em termos da função $\Psi(x, 0)$. Por exemplo, neste caso, podemos assumir que a função de onda é caracterizada como uma distribuição gaussiana em torno de $\langle x\rangle$ com desvio padrão $\sigma$ (ver expressão B6 do apêndice B).

$$
\Psi(x, 0)=\sigma^{-\frac{1}{2}} \pi^{-\frac{1}{4}} \mathrm{e}^{-\frac{1}{2}\left(\frac{x-\langle x\rangle}{\sigma}\right)^{2}} .
$$

Se considerarmos que o x médio da medida é a origem, então $\langle x\rangle=0$. Desta forma, (conforme Postulado 5 do apêndice A) a função densidade de probabilidade pode ser escrita como

$$
|\Psi(x, 0)|^{2}=\frac{1}{\sigma \sqrt{\pi}} e^{-\left(\frac{x}{\sigma}\right)^{2}} .
$$

e tem um desvio padrão $\sigma_{x}=\sigma / \sqrt{2}$. As constantes da equação (7) e (6) são determinadas de forma que a integral de $|\Psi(x, 0)|^{2}$ sobre todo espaço seja igual a 1 (propriedades da integral gaussiana estão no apêndice B). Podemos, agora, descrever $\Psi(x, 0)$ em termos da função de coeficiente $g(k)$

$$
\Psi(x, 0)=\frac{1}{2 \pi} \int_{-\infty}^{+\infty} g(k) e^{i k x} d k .
$$

É possível reconhecer que $g(k)$ é a Transformada de Fourier de $\Psi(x, 0)$ - por isso o termo $1 / 2 \pi$ foi introduzido em (5) (comparar, por exemplo, com as expressões B7 e B8 do apêndice B). Além disso, também é possível interpretar a equação acima como a decomposição espectral de $\Psi(x, 0)$ em termos dos autoestados do operador $\hat{\mathbf{k}}$. Podemos ainda reescrever a integral, com o objetivo de tornar o momentum da partícula explícito, por meio da seguinte mudança de variáveis

$$
k=\frac{p}{\hbar} .
$$

De modo que

$$
g(k)=g\left(\frac{p}{\hbar}\right)=\sqrt{\hbar} \psi(p) .
$$

E, portanto, é possível reescrever a expressão (8) em termos da variável $p$

$$
\Psi(x, 0)=\frac{1}{2 \pi \sqrt{\hbar}} \int_{-\infty}^{+\infty} \psi(p) e^{\frac{i p x}{\hbar}} d p .
$$

Calculando a transformada de Fourier (apêndice B), obtemos

$$
\psi(p)=\frac{1}{\sqrt{\hbar}} \int_{-\infty}^{+\infty} \Psi(x, 0) e^{-\frac{i p x}{\hbar}} d x
$$

que é a função de onda na representação de momentum, escrita como uma integração sobre os autoestados do operador $\widehat{\mathbf{x}}$. Usando $\Psi(x, 0)$ de (6) e substituindo em (12), temos

$$
\psi(p)=\frac{1}{\sqrt{\hbar}} \int_{-\infty}^{+\infty} \sigma^{-\frac{1}{2}} \pi^{-\frac{1}{4}} e^{-\frac{1}{2}\left(\frac{x-\langle x\rangle}{\sigma}\right)^{2}} e^{-\frac{i p x}{\hbar}} d x .
$$


Resolvendo a integral com $\langle x\rangle=0$, (ver apêndice B) obtemos:

$$
\psi(p)=\left(\frac{\sigma^{2} \pi}{\hbar^{2}}\right)^{\frac{1}{4}} e^{-\frac{\sigma^{2} p^{2}}{2 \hbar^{2}}} .
$$

Esta é uma nova função gaussiana, mas cujo desvio padrão é igual a $\hbar / \sigma$. Com isso podemos determinar a função densidade de probabilidade na representação de momentum:

$$
|\psi(p)|^{2}=\frac{\sigma \sqrt{\pi}}{\hbar} e^{-\frac{\sigma^{2} p^{2}}{\hbar^{2}}}
$$

Esta equação expressa a densidade de probabilidade para medir certo valor de momentum para a partícula. Neste caso, é possível determinar que o desvio padrão da função de densidade é $\sigma_{p}=\hbar /(\sigma \sqrt{2}$ ) (conforme apêndice B).

Finalmente, podemos calcular que o produto entre o desvio padrão da função de densidade de probabilidade nas representações posição e momentum é

$$
\sigma_{x} \cdot \sigma_{p}=\frac{\sigma}{\sqrt{2}} \cdot \frac{\hbar}{\sigma \sqrt{2}}=\frac{\hbar}{2} .
$$

Podemos observar que Heisenberg encontra uma igualdade e não uma desigualdade. Isso acontece porque calculamos a relação de incerteza para o caso de uma função gaussiana, o que corresponde justamente ao pacote de menor incerteza possível [52]. Assim, a derivação feita por Heisenberg caracteriza apenas um caso particular e não é uma demonstração geral da relação de incerteza entre posição e momentum. Ademais, ressaltamos que Heisenberg não relaciona diretamente a relação de incerteza com o comutador dos operadores conforme ele mesmo anuncia em seu artigo. Isso somente é feito em derivações futuras - como discutiremos mais adiante.

\subsection{Incerteza entre as variáveis posição e momentum para um pacote de ondas generalizado - Derivação de Weyl (1928)}

Uma outra maneira de demonstrar o Princípio da Incerteza é por meio da derivação proposta por Herman Weyl em 1928 [53. Ao contrário da demonstração anterior, proposta por Heisenberg e exclusivamente válida para funções gaussianas, a estratégia utilizada por Weyl independe do formato da função de onda, e, portanto, podemos dizer que é para um pacote generalizado. Para isso, Weyl utiliza o fato de que observáveis são operadores Hermitianos e, portanto, (conforme apêndice B) para um observável arbitrário $\widehat{\mathbf{A}}$, podemos escrever sua variância como o valor médio

$$
\sigma_{A}^{2}=\left\langle(\widehat{\mathbf{A}}-\langle\widehat{\mathbf{A}}\rangle)^{2}\right\rangle=\left\langle\widehat{\mathbf{A}}^{2}\right\rangle-\langle\widehat{\mathbf{A}}\rangle^{2} .
$$

Que pode ser calculado como

$$
\sigma_{A}^{2}=\int \Psi^{*}\left(\widehat{\mathbf{A}}^{2}-\langle\widehat{\mathbf{A}}\rangle^{2}\right) \Psi d x .
$$

E, além disso, Weyl utiliza o fato de que o operador momentum, especificamente, pode ser escrito como

$$
p=-i \hbar \frac{\partial}{\partial x} \text {. }
$$

Consideramos, então, uma partícula na posição $x$. De modo que, por simplicidade, o valor médio da posição é 0. Então

$$
\sigma_{x}^{2}=\int \Psi^{*}\left(x^{2}-\langle 0\rangle^{2}\right) \Psi d x
$$

Ou seja:

$$
\sigma_{x}^{2}=\int \Psi^{*} x^{2} \Psi d x
$$

É possível proceder da mesma maneira para o momentum da partícula. Dessa forma, considerando, por simplicidade, $\langle p\rangle=0$, podemos escrever

$$
\sigma_{p}^{2}=\int \Psi^{*} p^{2} \Psi d x
$$

Lembrando, ainda, que é possível expressar o operador momentum como $p=-i \hbar \partial / \partial x$, temos

$$
\begin{aligned}
\sigma_{p}^{2} & =\int \Psi^{*}\left(-i \hbar \frac{\partial}{\partial x}\right)^{2} \Psi d x=\int\left(\frac{i \hbar \partial}{\partial x} \Psi\right)^{*}\left(\frac{-i \hbar \partial}{\partial x} \Psi\right) d x \\
& =-\hbar^{2} \int \frac{\partial \Psi^{*}}{\partial x} \frac{\partial \Psi}{\partial x} d x .
\end{aligned}
$$

Também é possível utilizar o fato de que $p$ é um operador Hermitiano. Tomando a desigualdade de Schwarz (conforme apêndice C)

$$
\begin{gathered}
\left\{\left[\int\left(f_{1} f_{1}^{*}+f_{2} f_{2}^{*}\right) d x\right] \cdot\left[\int\left(g_{1} g_{1}^{*}+g_{2} g_{2}^{*}\right) d x\right]\right\} \\
\geq\left|\int f_{1} g_{1} d x+\int f_{2} g_{2} d x\right|^{2}
\end{gathered}
$$

e substituindo $f_{1}=x \Psi=f_{2}^{*}$ e $g_{1}=\frac{\partial \Psi^{*}}{\partial x}=g_{2}^{*}$ no lado esquerdo da expressão (24), temos

$$
\begin{aligned}
{\left[\int\left(x \Psi x \Psi^{*}+x \Psi^{*} x \Psi\right) d x\right] } \\
\cdot\left[\int\left(\frac{\partial \Psi^{*}}{\partial x} \frac{\partial \Psi}{\partial x}+\frac{\partial \Psi}{\partial x} \frac{\partial \Psi^{*}}{\partial x}\right) d x\right],
\end{aligned}
$$

o que resulta em

$$
4\left[\int \Psi x^{2} \Psi^{*} d x\right] \cdot\left[\int \frac{\partial \Psi}{\partial x} \frac{\partial \Psi^{*}}{\partial x} d x\right] .
$$

Substituindo as expressões (21) e (22) em (26), obtemos que o lado esquerdo da expressão (24) pode ser escrito como

$$
\frac{4 \sigma_{x}^{2} \sigma_{p}^{2}}{\hbar^{2}}
$$


Já no lado direito da expressão 24, temos que

$$
\begin{aligned}
\left|\int f_{1} g_{1} d x+\int f_{2} g_{2} d x\right|^{2} \\
=\left|\int x \Psi \frac{\partial \Psi^{*}}{\partial x} d x+\int x \Psi^{*} \frac{\partial \Psi}{\partial x} d x\right|^{2} \\
=\left|\int \frac{x \partial}{\partial x}\left(\Psi^{*} \Psi\right) d x\right|^{2} .
\end{aligned}
$$

Fazendo a integração por partes, $\int u d v=u . v-\int v d u$, escolhendo $u=x$ e $d v=\partial / \partial x\left(\Psi^{*} \Psi\right) d x$, obtemos

$$
\int x \frac{\partial}{\partial x}\left(\Psi^{*} \Psi\right) d x=\left.x \cdot \Psi^{*} \Psi\right|_{-\infty} ^{+\infty}-\int \Psi^{*} \Psi d x=-1 .
$$

Assim, para que possa ter significado físico, a expressão $\left(x \cdot \Psi^{*} \Psi\right)$ deve tender a zero para $|x| \rightarrow \infty$. Portanto,

$$
\left|\int x \frac{\partial}{\partial x}\left(\Psi^{*} \Psi\right) d x\right|^{2}=|-1|^{2} .
$$

Obtemos então a desigualdade

$$
\frac{4 \sigma_{x}^{2} \sigma_{p}^{2}}{\hbar^{2}} \geq|-1|^{2} .
$$

E, portanto,

$$
\sigma_{x} \sigma_{p} \geq \frac{\hbar}{2}
$$

Diferentemente da derivação de Heisenberg, que determina a relação de incerteza entre posição e momentum apenas para o caso gaussiano, com a derivação de Weyl, temos a relação para quaisquer formatos de pacote de onda.

\subsection{Incerteza entre dois observáveis quaisquer A e B - Derivação de Robertson (1929)}

Até esse momento, apenas a expressão do Princípio da Incerteza para as variáveis posição e momentum havia se mostrado consistente com o formalismo da Mecânica Quântica. Entretanto, como foi discutido anteriormente, Heisenberg havia afirmado que todas as variáveis canonicamente conjugadas deveriam obedecer a tal relação. A prova matemática para isso só veio em 1929 no artigo publicado por Robertson [54. Neste artigo, Robertson utiliza a expressão para a desigualdade de Schwarz, assim como proposto por Weyl, para relacionar o quadrado do desvio padrão de dois observáveis representados por operadores Hermitianos. Portanto, (conforme apêndice B) para um observável arbitrário $\widehat{\mathbf{A}}$, podemos escrever sua variância como

$$
\sigma_{A}^{2}=\left\langle(\widehat{\mathbf{A}}-\langle\widehat{\mathbf{A}}\rangle \widehat{\mathbf{I}})^{2}\right\rangle=\left\langle\widehat{\mathbf{A}}^{2}\right\rangle-\langle\widehat{\mathbf{A}}\rangle^{2}=\left\langle\delta \widehat{\mathbf{A}}^{2}\right\rangle,
$$

sendo $\delta \widehat{\mathbf{A}}=\widehat{\mathbf{A}}-\langle\widehat{\mathbf{A}}\rangle \widehat{\mathbf{I}}$, sendo $\widehat{\mathbf{I}}$ o operador identidade (evidentemente $\langle\widehat{\mathbf{I}}\rangle=1$ ). A variância pode ser calculada como

$$
\sigma_{A}^{2}=\int \Psi^{*}\left(\widehat{\mathbf{A}}^{2}-\langle\widehat{\mathbf{A}}\rangle^{2}\right) \Psi d x
$$

Considerando que para dois observáveis quaisquer $(\widehat{\mathbf{A}}$ e $\widehat{\mathbf{B}})$, podemos escrever suas variâncias, respectivamente, como

$$
\begin{aligned}
\sigma_{A}^{2} & =\left\langle\delta \widehat{\mathbf{A}}^{2}\right\rangle=\int \Psi^{*}\left(\widehat{\mathbf{A}}^{2}-\langle\widehat{\mathbf{A}}\rangle^{2}\right) \Psi d x, \\
\sigma_{B}^{2} & =\left\langle\delta \widehat{\mathbf{B}}^{2}\right\rangle=\int \Psi^{*}\left(\widehat{\mathbf{B}}^{2}-\langle\widehat{\mathbf{B}}\rangle^{2}\right) \Psi d x .
\end{aligned}
$$

Agora, tomando as expressões $f_{1}=(\widehat{\mathbf{A}}-\langle\widehat{\mathbf{A}}\rangle) \Psi^{*}, f_{2}=$ $(\widehat{\mathbf{A}}-\langle\widehat{\mathbf{A}}\rangle) \Psi, g_{1}=(\widehat{\mathbf{B}}-\langle\widehat{\mathbf{B}}\rangle) \Psi$ e $g_{2}=-(\widehat{\mathbf{B}}-\langle\widehat{\mathbf{B}}\rangle) \Psi^{*} \mathrm{e}$ substituindo na desigualdade de Schwarz (24), no lado direito da inequação temos

$$
\left[2 \int \Psi^{*}\left(\widehat{\mathbf{A}}^{2}-\langle\widehat{\mathbf{A}}\rangle^{2}\right) \Psi d x\right] \cdot\left[2 \int \Psi^{*}\left(\widehat{\mathbf{B}}^{2}-\langle\widehat{\mathbf{B}}\rangle^{2}\right) \Psi d x\right] .
$$

De modo que, levando em conta as expressões para as variâncias $\Delta \widehat{\mathbf{A}}$ e $\Delta \widehat{\mathbf{B}}$ dadas pelas expressões 35 e $(36)$, obtemos:

$$
\begin{aligned}
4\left[\int\right. & \left.\Psi^{*}\left(\widehat{\mathbf{A}}^{2}-\langle\widehat{\mathbf{A}}\rangle^{2}\right) \Psi d x\right] \cdot\left[\int \Psi^{*}\left(\widehat{\mathbf{B}}^{2}-\langle\widehat{\mathbf{B}}\rangle^{2}\right) \Psi d x\right] \\
& =4 \sigma_{A}^{2} \sigma_{B}^{2} \\
& =4\left\langle\delta \widehat{\mathbf{A}}^{2}\right\rangle\left\langle\delta \widehat{\mathbf{B}}^{2}\right\rangle
\end{aligned}
$$

Substituindo as expressões para $f$ e $g$ no lado esquerdo da desigualdade de Schwarz (24), temos

$$
\begin{aligned}
& \mid \int(\widehat{\mathbf{A}}-\langle\widehat{\mathbf{A}}\rangle) \Psi^{*}(\widehat{\mathbf{B}}-\langle\widehat{\mathbf{B}}\rangle) \Psi d x \\
& \quad+\left.\int(\widehat{\mathbf{A}}-\langle\widehat{\mathbf{A}}\rangle) \Psi(\widehat{\mathbf{B}}-\langle\widehat{\mathbf{B}}\rangle) \Psi^{*} d x\right|^{2} .
\end{aligned}
$$

Abrindo os termos da expressão 39 , e utilizando o fato de que $\int \Psi^{*} \widehat{\mathbf{A}} \Psi d x=\int \Psi \widehat{\mathbf{A}}^{\dagger} \Psi^{*} d x$ temos que o lado direito da expressão (24) resulta em

$$
\left|\int \Psi^{*}(\widehat{\mathbf{A}} \widehat{\mathbf{B}}-\widehat{\mathbf{B}} \widehat{\mathbf{A}}) \Psi d x\right|^{2} \text {. }
$$

A relação $\widehat{\mathbf{A}} \widehat{\mathbf{B}}-\widehat{\mathbf{B}} \widehat{\mathbf{A}}$ pode ser expressa como $[\widehat{\mathbf{A}}, \widehat{\mathbf{B}}]$ (operação de comutação entre os observáveis $\widehat{\mathbf{A}}$ e $\widehat{\mathbf{B}}$ ). Unindo as expressões (38) e 40 na expressão (24), obtemos

$$
4 \sigma_{A}^{2} \sigma_{B}^{2}=4\left\langle(\delta \widehat{\mathbf{A}})^{2}\right\rangle\left\langle(\delta \widehat{\mathbf{B}})^{2}\right\rangle \geq|\langle[\widehat{\mathbf{A}}, \widehat{\mathbf{B}}]\rangle|^{2} .
$$

Então

$\sigma_{A} \sigma_{B}=\sqrt{\left\langle(\delta \widehat{\mathbf{A}})^{2}\right\rangle\left\langle(\delta \widehat{\mathbf{B}})^{2}\right\rangle}=\langle\Delta \widehat{\mathbf{A}}\rangle\langle\Delta \widehat{\mathbf{B}}\rangle \geq \frac{1}{2}|\langle[\widehat{\mathbf{A}}, \widehat{\mathbf{B}}]\rangle|$.

$\mathrm{Na}$ expressão acima, definimos as dispersões médias dos observáveis $\widehat{\mathbf{A}}$ e $\widehat{\mathbf{B}}$ respectivamente como $\langle\Delta \widehat{\mathbf{A}}\rangle=$ $\sqrt{\left\langle(\delta \widehat{\mathbf{A}})^{2}\right\rangle}$ e $\langle\Delta \widehat{\mathbf{B}}\rangle=\sqrt{\left\langle(\delta \widehat{\mathbf{B}})^{2}\right\rangle}$. Assim, a afirmação de Heisenberg sobre a conexão entre as relações de incerteza e a propriedade de comutação foi finalmente provada. 
Assim, podemos concluir que para dois observáveis que não comutam, as suas respectivas variâncias são relacionadas por meio de uma desigualdade que depende do comutador entre esses mesmos observáveis. A relação (42) mostra que um sistema não pode ser preparado em um estado quântico tal que duas grandezas físicas cujos observáveis que as representam não comutam entre si sejam livres de dispersão. Uma dispersão baixa em uma das grandezas (baixa incerteza) acarreta uma dispersão alta na outra (incerteza alta). Se os observáveis comutam, essa limitação não existe.

\subsection{A derivação do princípio da incerteza por Schrödinger (1930)}

Erwin Schrödinger [55] também parte da desigualdade de Schwarz (apêndice C), mas neste caso, escrita por meio da expressão simplificada

$$
\int f f^{*} d x \cdot \int g g^{*} d x \geq\left|\int f g d x\right|^{2}
$$

Entretanto, o passo que diferencia a derivação de Schrödinger da derivação de Robertson é o fato de que Schrödinger propõe escrever o produto de dois operadores (supostos hermitianos) como uma soma simétrica

$$
\widehat{\mathbf{A}} \widehat{\mathbf{B}}=\frac{\widehat{\mathbf{A}} \widehat{\mathbf{B}}+\widehat{\mathbf{B}} \widehat{\mathbf{A}}}{2}+\frac{\widehat{\mathbf{A}} \widehat{\mathbf{B}}-\widehat{\mathbf{B}} \widehat{\mathbf{A}}}{2}
$$

Agora, tomando $f=\widehat{\mathbf{B}} \Psi$ e $g=\widehat{\mathbf{A}}^{\dagger} \Psi^{*}$, podemos escrever

$$
\int \widehat{\mathbf{B}} \Psi \widehat{\mathbf{B}}^{\dagger} \Psi^{*} d x . \int \widehat{\mathbf{A}}^{\dagger} \Psi^{*} \widehat{\mathbf{A}} \Psi d x \geq\left|\int \widehat{\mathbf{B}} \Psi \widehat{\mathbf{A}}^{\dagger} \Psi^{*} d x\right|^{2} .
$$

De modo que, seguindo [56], temos

$$
\begin{aligned}
\int \Psi \widehat{\mathbf{B}}^{2} \Psi^{*} d x \cdot \int \Psi^{*} \widehat{\mathbf{A}}^{2} \Psi d x \\
\geq\left|\int \Psi \widehat{\mathbf{A}} \widehat{\mathbf{B}} \Psi^{*} d x\right|^{2} \\
=\left(\int \Psi \widehat{\mathbf{A}} \widehat{\mathbf{B}} \Psi^{*} d x\right)\left(\int \Psi^{*} \widehat{\mathbf{B}} \widehat{\mathbf{A}} \Psi d x\right) .
\end{aligned}
$$

No lado direito de (46) foi usada a propriedade $(\widehat{\mathbf{A}} \widehat{\mathbf{B}})^{\dagger}=\widehat{\mathbf{B}}^{\dagger} \widehat{\mathbf{A}}^{\dagger}=\widehat{\mathbf{B}} \widehat{\mathbf{A}}$. Supondo, sem perda de generalidade 8 que $\langle\widehat{\mathbf{A}}\rangle=\langle\widehat{\mathbf{B}}\rangle=0$, temos

$$
\sigma_{A}^{2}=\left\langle\widehat{\mathbf{A}}^{2}\right\rangle=\int \Psi^{*} \widehat{\mathbf{A}}^{2} \Psi d x
$$

e

$$
\sigma_{B}^{2}=\left\langle\widehat{\mathbf{B}}^{2}\right\rangle=\int \Psi^{*} \widehat{\mathbf{B}}^{2} \Psi d x
$$

8 Qualquer operador $\widehat{\mathbf{O}}$ pode ser reescrito em outro operador $\widehat{\mathbf{O}}^{\prime}$ cujo valor esperado é nulo. Basta simplesmente fazer $\widehat{\mathbf{O}}^{\prime}=\widehat{\mathbf{O}}-$ $\langle\widehat{\mathbf{O}}\rangle \widehat{\mathbf{I}}$. Nesse caso, por construção, $\left\langle\widehat{\mathbf{O}}^{\prime}\right\rangle=0$.
Então,

$$
\langle\widehat{\mathbf{A}} \widehat{\mathbf{B}}\rangle\langle\widehat{\mathbf{B}} \widehat{\mathbf{A}}\rangle=\left|\int \Psi A B \Psi^{*} d x\right|^{2} .
$$

Temos

$$
\left\langle\widehat{\mathbf{A}}^{2}\right\rangle\left\langle\widehat{\mathbf{B}}^{2}\right\rangle \geq\langle\widehat{\mathbf{A}} \widehat{\mathbf{B}}\rangle\langle\widehat{\mathbf{B}} \widehat{\mathbf{A}}\rangle
$$

Escrevendo $\langle\widehat{\mathbf{A}} \widehat{\mathbf{B}}\rangle\langle\widehat{\mathbf{B}} \widehat{\mathbf{A}}\rangle$ em termos da soma simétrica dada por (44), obtemos

$$
\left\langle\widehat{\mathbf{A}}^{2}\right\rangle\left\langle\widehat{\mathbf{B}}^{2}\right\rangle \geq \frac{1}{4}(\langle\widehat{\mathbf{A}} \widehat{\mathbf{B}}+\widehat{\mathbf{B}} \widehat{\mathbf{A}}\rangle)^{2}-\frac{1}{4}(\langle\widehat{\mathbf{A}} \widehat{\mathbf{B}}-\widehat{\mathbf{B}} \widehat{\mathbf{A}}\rangle)^{2}
$$

Usando a propriedade $|\langle\widehat{\mathbf{A}} \widehat{\mathbf{B}}-\widehat{\mathbf{B}} \widehat{\mathbf{A}}\rangle|^{2}=-(\langle\widehat{\mathbf{A}} \widehat{\mathbf{B}}-$ $\widehat{\mathbf{B}} \widehat{\mathbf{A}}\rangle)^{2}$, a expressão 51 pode ser reescrita como

$$
\left\langle\widehat{\mathbf{A}}^{2}\right\rangle\left\langle\widehat{\mathbf{B}}^{2}\right\rangle \geq \frac{1}{4}(\langle\widehat{\mathbf{A}} \widehat{\mathbf{B}}+\widehat{\mathbf{B}} \widehat{\mathbf{A}}\rangle)^{2}+\frac{1}{4}|\langle\widehat{\mathbf{A}} \widehat{\mathbf{B}}-\widehat{\mathbf{B}} \widehat{\mathbf{A}}\rangle|^{2},
$$

Definindo o anticomutador $\{\widehat{\mathbf{A}}, \widehat{\mathbf{B}}\}=\widehat{\mathbf{A}} \widehat{\mathbf{B}}+\widehat{\mathbf{B}} \widehat{\mathbf{A}}$, e lembrando que $[\widehat{\mathbf{A}}, \widehat{\mathbf{B}}]=\widehat{\mathbf{A}} \widehat{\mathbf{B}}-\widehat{\mathbf{B}} \widehat{\mathbf{A}}$, substituindo as expressões 47) e 48) em (52), obtemos

$$
\sigma_{A}^{2} \sigma_{B}^{2} \geq \frac{1}{4}\langle\{\widehat{\mathbf{A}}, \widehat{\mathbf{B}}\}\rangle^{2}+\frac{1}{4}|\langle[\widehat{\mathbf{A}}, \widehat{\mathbf{B}}]\rangle|^{2}=\sigma_{A B}^{2}+\frac{1}{4}|\langle[\widehat{\mathbf{A}}, \widehat{\mathbf{B}}]\rangle|^{2} .
$$

O segundo termo no lado direito da equação é igual à expressão de Robertson e o primeiro termo no lado direito pode ser reconhecido como a covariância $\sigma_{A B}=(1 / 2)\langle\{\delta \widehat{\mathbf{A}}, \delta \widehat{\mathbf{B}}\}\rangle=(1 / 2)\langle\{\widehat{\mathbf{A}}, \widehat{\mathbf{B}}\}\rangle-\langle\widehat{\mathbf{A}}\rangle\langle\widehat{\mathbf{B}}\rangle=$ $(1 / 2)\langle\{\widehat{\mathbf{A}}, \widehat{\mathbf{B}}\}\rangle$ entre os observáveis $\widehat{\mathbf{A}}$ e $\widehat{\mathbf{B}}$ (lembrando que seus respectivos valores esperados são nulos por hipótese) [57, 58. No caso em que os valores esperados dos observáveis $\widehat{\mathbf{A}}$ e $\widehat{\mathbf{B}}$ não são nulos, é fácil mostrar que basta inserir em (53) a expressão completa para $\sigma_{A B}{ }^{9}$ Nesse sentido, pode-se entender que a Incerteza de Heisenberg é um caso especial de Incerteza de Schrödinger, no qual não se leva em conta a covariância entre as duas observáveis.

Por fim, é importante notar que o primeiro termo do lado direito da equação 53 é estritamente positivo (limite inferior), e isso, por si só, já é suficiente para provar a relação de incerteza conhecida quando os operadores envolvidos não comutam (quantidade positiva bem como na equação (53) - o primeiro termo só pode aumentar a contribuição do segundo termo). Como a propriedade importante é, se o produto de $\Delta x \Delta p$ der um número positivo, diferente de zero, temos o que precisamos: se a precisão no conhecimento de um aumenta, a precisão do outro deve diminuir, e vice-versa.

9 A variância $\sigma_{A}^{2}$ nada mais é do que um caso particular da covariância, podendo ser entendida como a covariância do observável $\widehat{\mathbf{A}}$ consigo mesmo. Basta fazer $\sigma_{A}^{2} \equiv \sigma_{A A}=(1 / 2)\langle\{\delta \widehat{\mathbf{A}}, \delta \widehat{\mathbf{A}}\}\rangle=$ $\left\langle\delta \widehat{\mathbf{A}}^{2}\right\rangle=\left\langle\widehat{\mathbf{A}}^{2}\right\rangle-\langle\widehat{\mathbf{A}}\rangle^{2}$. 


\section{Segundo Nível do Princípio da Incerteza: Fenomenologia}

Na Teoria Quântica o processo de medição está diretamente associado ao colapso da função de onda (Apêndice A). Se, por exemplo, sabemos que uma partícula passou por uma fenda então, naquele instante, o pacote de ondas deve ter colapsado para o tamanho da fenda e, portanto, de acordo com a transformada de Fourier, a distribuição do momentum não poderá ser representada por um único valor (devendo ser, então, uma distribuição de valores). Entretanto, propomos que nesta seção o estudo dos experimentos propostos por Werner Heisenberg permaneça apenas a nível fenomenológico, de modo que algumas das possíveis interpretações de tais fenômenos subatômicos serão discutidas no próximo nível.

\subsection{O Microscópio de raios Gamma}

No artigo de 1927 o primeiro argumento utilizado para justificar o Princípio da Incerteza também é o primeiro experimento mental proposto por Heisenberg, que ficou conhecido como o Experimento do Microscópio. Nessa análise fenomenológica, Heisenberg discute a possibilidade de determinar a posição de um elétron utilizando um microscópio [1].

Por exemplo, vamos iluminar o elétron e observá-lo ao microscópio. Então, a maior precisão alcançável na medição da posição é governada pelo comprimento de onda da luz. No entanto, em princípio, pode-se construir, digamos, um microscópio de raios gama e, com ele, realizar a determinação da posição com a precisão desejada. Nesta medição, há um recurso importante, o efeito Compton. Toda observação de luz espalhada proveniente do elétron pressupõe um efeito fotoelétrico (no olho, um no plano fotográfico, na fotocélula) e, portanto, também pode ser interpretada de forma que um quantum de luz atinge o elétron, é refletido ou espalhado, e então, mais uma vez dobrado pela lente do microscópio, produz o efeito fotoelétrico. No instante em que as posições são determinadas - portanto, no momento em que o fóton é espalhado pelo elétron o elétron sofre uma mudança descontínua no momento. Essa mudança é tanto maior quanto menor for o comprimento de onda da luz empregada - ou seja, mais exata será a determinação da posição. No instante em que a posição do elétron é conhecida, seu momento, portanto, pode ser conhecido em magnitudes que correspondem a essa mudança descontínua. Assim, quanto mais precisamente a posição é determinada, menos precisamente o momento é conhecido e viceversa. Nesta circunstância, vemos uma interpretação física direta da equação $p q-q p=$ $-i \hbar$. Seja $q_{1}$ a precisão com a qual o valor $q$ é conhecido ( $q_{1}$ é, digamos, o erro médio de $q$ ), portanto aqui o comprimento de onda da luz. Seja $p_{1}$ a precisão com a qual o valor $p$ é determinável; isto é, aqui, a mudança descontínua de $p$ no efeito Compton. Então, de acordo com as leis elementares do efeito Compton, $p_{1}$ e $q_{1}$ estão na relação $p_{1} q_{1} \sim h$ (1983, p. 64, tradução livre) [1].

Uma simplificação corrigida do experimento de Heisenberg é proposta por [18] e está representada na Figura 1

Suponhamos que um feixe de fótons de raios gamma está inicialmente ao longo do eixo da lente do microscópio. Supondo que a lente possui um diâmetro $D$, a resolução angular $\delta \theta$ pode ser descrita como

$$
\delta \theta \approx \frac{\lambda}{D}
$$

Tomando $F$ como a distância focal da lente, temos que o objeto está a uma distância $\delta q$ descrita por

$$
\delta q \approx F \delta \theta \approx \frac{\lambda F}{D}
$$

O fóton originalmente possui um momentum $p_{\gamma}$ que, após ser elasticamente espalhado pela lente, adquire um componente transversal no intervalo compreendido entre $-p_{\gamma} \mathrm{e}+p_{\gamma}$. Além disso, para ângulos pequenos, temos que

$$
\operatorname{sen} \phi \approx \tan \phi=\frac{D}{2 F}
$$

Dessa forma, a incerteza no componente transversal do momentum do fóton será

$$
p_{\gamma} \approx\left(\frac{D}{F}\right) p_{\gamma} \approx \frac{h D}{\lambda F}
$$

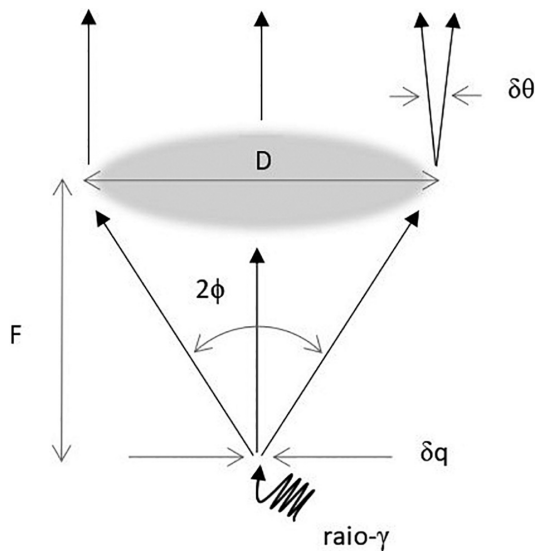

Figura 1: Representação esquematizada do microscópio de raios gamma. 
e pela conservação do momentum, a incerteza do momentum do elétron torna-se

$$
\delta p \approx \frac{h D}{\lambda F}
$$

De modo que, multiplicando as equações (55) e (58), temos a relação de incerteza de Heisenberg

$$
\delta q \delta p \sim h
$$

\subsection{Um elétron passando por uma fenda}

Outro experimento mental a qual Heisenberg faz referência ao longo do texto de 1927 é o de um elétron atravessando uma fenda. O fenômeno consiste em fazer com que um elétron atravesse uma pequena abertura, seja ela um orifício ou uma fenda, de maneira que a onda associada ao movimento do elétron sofrerá o efeito de difração, resultando na alteração de sua trajetória. Dessa forma, a partir do momento em que o elétron atravessa a fenda, torna-se impossível prever exatamente o local em que o mesmo será detectado na chapa fotográfica [59].

\begin{abstract}
"Esta deflexão deve ser de pelo menos a mesma ordem de magnitude que o alargamento natural do feixe provocado pela difração pelas fendas, se qualquer medição for possível. O ângulo de difração é aproximadamente $\lambda / d$ se $\lambda$ denota o comprimento de onda de Broglie; portanto, $\lambda / d \sim E_{1} t_{1} d p$, ou como $\lambda=h / p, E_{1} t_{1} \sim h . "(1983$, p. 67 , tradução livre) [1].
\end{abstract}

Para calcular a incerteza associada ao experimento é preciso ter em vista que, antes de atravessar o diafragma, o estado de movimento da partícula pode ser representado por uma série de ondas planas de comprimento de onda $\lambda$, e seu momentum $p$ é associado a $\lambda$ segundo a relação de de Broglie:

$$
p=\frac{h}{\lambda}
$$

Entretanto, após a passagem pelo orifício, a onda associada ao movimento da partícula sofre o efeito de difração e, além disso, é possível interpretar que a partícula adquire um momentum em sua componente paralela à extensão da fenda $\left(p_{y}\right)$. Tendo em vista que o padrão de distribuição gerado na chapa fotográfica por um conjunto de partículas incidindo sucessivamente por uma fenda é muito semelhante a um padrão de interferência, tal padrão pode ser determinado por meio de uma descrição ondulatória. Quando se incide luz coerente, a franja de máximo central concentra quase toda a energia luminosa, de modo que podemos considerar analogamente que a probabilidade de detecção da partícula se concentra quase que totalmente nessa região. Para determinar a largura dessa região basta calcular a posição da primeira franja de mínimo, dada pela equação (61) 60]:

$$
\operatorname{arcsen}(\theta)=\lambda
$$

em que $a$ é a largura da fenda e $\theta$ é a posição angular do mínimo. Expandindo a função $\operatorname{sen}(\theta)$ em séries de Taylor podemos considerar $\operatorname{sen}(\theta) \approx \theta$. Usando tal aproximação na equação 61 obtemos

$$
\theta \approx \frac{\lambda}{a}
$$

A incerteza associada ao momentum adquirido pela partícula $\Delta p$ está, também, associada à largura do máximo central, de modo que quanto maior for o momentum $p$ adquirido, maior será o desvio da sua trajetória e, portanto, mais próxima às extremidades dessa região a partícula será detectada. Dessa forma, a incerteza $\Delta p$ corresponde ao maior valor que pode ser adquirido por $p$ em sua componente $p_{y}$. Relacionando o módulo de ambos os vetores através de uma análise trigonométrica, temos

$$
\operatorname{sen} \theta=\frac{p_{y}}{p}
$$

de modo que

$$
p_{y} \approx \theta p
$$

e podemos descrever $\Delta p$ como

$$
\Delta p=p_{y m a ́ x}=\theta p
$$

substituindo as equações (60) e 62 na equação 65 :

$$
\Delta p \approx \frac{h}{a}
$$

Além da incerteza no momentum, existe também uma incerteza $\Delta q$ na medida da posição da partícula no instante em que atravessa a fenda. Sabemos que a partícula passa pela fenda, mas não sabemos em que ponto exato da extensão da fenda ela está localizada. Já que os limites em que é possível encontrar a partícula correspondem ao espaço no interior da fenda, podemos definir $\Delta q$ como sendo a própria largura a da fenda, de modo que

$$
\Delta p \approx \frac{h}{\Delta q}
$$

Reorganizando a expressão (67), obtemos a equação 68 nos mostra que as incertezas associadas estão de acordo com a relação de indeterminação.

$$
\Delta p \Delta q \approx h
$$

\section{Terceiro Nível do Princípio da Incerteza: Interpretações do Princípio da Incerteza para as Variáveis Posição e Momentum}

O terceiro nível, que diz respeito interpretação de uma teoria ou conceito físico, está sempre na fronteira entre 
a Física e a Filosofia. É possível que, em cursos de graduação, possa-se evitar o terceiro nível, quando o foco da disciplina está mais voltado para o desenvolvimento do domínio do formalismo matemático. Entretanto, cursos voltados a abordagens históricas e conceituais podem ser mais favoráveis à sua incorporação de maneira integral à teoria, tal como Pessoa Jr. 34.

Apresentamos aqui quatro interpretações que a literatura aponta para o Princípio da Incerteza, que podem ser discutidas por professores que tenham interesse em trazer a dimensão interpretativa da Teoria para o ensino. Por outro lado, aqueles e aquelas que perceberem nessa seção aspectos que fujam do escopo da Física podem optar por não a utilizar.

Depois de mais de 90 anos da primeira apresentação do Princípio da Incerteza de Heisenberg uma ampla literatura foi desenvolvida com o objetivo de abordar seus diferentes significados e implicações [61]. Ao longo desta seção, iremos seguir a discussão e classificação proposta por Jijnasu 23. Rigorosamente, as interpretações utilizadas para a incerteza nas variáveis posição e momentum podem ser divididas entre versões ônticas e versões epistêmicas. As versões do tipo ônticas são aquelas que propõe que a incerteza é uma característica inerente da matéria, independentemente de qualquer medida, enquanto as versões do tipo epistêmicas propõem que a incerteza é uma característica do procedimento de medida, embora, ainda assim, não possa ser evitada. Cabe destacar que a formulação original de Heisenberg mistura ambas concepções. Ao longo do artigo de 1927, na apresentação do experimento do microscópio de raiosgama e durante a derivação do Princípio da Incerteza utilizando o formalismo de Dirac-Jordan, Heisenberg assume uma postura epistêmica - atando as incertezas a medição. Entretanto, ao final do artigo, Heisenberg assume uma inclinação positivista e interpreta todos os resultados como se a indeterminação fosse uma característica intrínseca da matéria.

Definimos como perspectiva ôntica aquilo que Jijnasu 23] chama de perspectiva "incerteza intrínseca" (e designa pela letra grega $\Delta$ ). A visão de uma "realidade não definida" foi posteriormente recuperada por Heisenberg [50] com o conceito de potencialidade, que, por sua vez, deu origem à Física Aristotélica ${ }^{10}$ Além disso, em 1935 Popper 63 discutiu o fato de que as relações de Heisenberg poderiam ser interpretadas estatisticamente, isto é, o Princípio da Incerteza não trataria de eventos singulares, mas de dispersões de conjuntos. Nesta concepção, não podemos falar de qualquer limitação da precisão em uma única medição. Em princípio, poderíamos medir a posição e o momentum com qualquer precisão arbitrária. O que não podemos fazer é preparar um conjunto tão nitidamente definido em posição e momentum quanto desejamos. Tal visão também foi

\footnotetext{
10 Este conceito também foi proposto recentemente por Busch e Jaeger 62 no âmbito das Medidas de Operador Positivo (valorizado) (POMs).
}

endossada por interpretações mais contemporâneas da Mecânica Quântica [55] e pode ser considerada, também, como uma perspectiva ôntica, uma vez que reconhece as relações de indeterminação como uma característica natural da matéria (independentemente das medidas), e difere do primeiro caso, porém, por não considerar que elas possam ser aplicadas a uma única medição. Jijnasu [23] a chama de perspectiva "estatística" (e ele a designa pela letra grega $\sigma$ ). Os adeptos da interpretação da indeterminação intrínseca entendem que a "interpretação do conjunto" apenas descreve como a realidade "não afiada" pode se manifestar nas medições, sendo a manifestação pragmática da incerteza intrínseca [64.

Além disso, a interpretação estatística não impõe qualquer restrição de precisão ou exatidão em medições simultâneas. No entanto, os experimentos mentais de Heisenberg indicam que algum tipo de restrição pode acontecer (embora o formalismo que ele desenvolveu não tenha sido capaz de demonstrar isso). Motivados por esse problema, muitos estudos têm sido desenvolvidos a fim de determinar a restrição em medidas simultâneas - que são classificadas como versões epistêmicas. Jijnasu [23] divide essas versões em dois grupos: o primeiro trata da relação entre a precisão de uma medida e a perturbação que essa medida causa na variável conjugada, como faz Heisenberg no caso do microscópio de raios gama [59]. Nesse caso, o momentum do elétron não foi medido apenas a posição foi medida - e sobre o momentum foi apenas inferida sua faixa de possível perturbação. Jijnasu [23] chama essa perspectiva de incerteza de distúrbio de erro (e ele a designa por $\varepsilon-\eta$ ), sendo possível encontrar muitas críticas à versão perturbação [30, 60, 61 Ademais, em vez de relacionar o resultado de uma única medição com o distúrbio que ela causa, também é possível realizar uma segunda medição para determinar o momentum do elétron. Nesse caso, também é possível provar que as incertezas entre o erro de posição e o erro de momentum obedecem a uma relação de incerteza [7, 61 63], Jijnasu [23] denomina tal perspectiva de incerteza como erro-erro (e ele a designa por $\varepsilon-\varepsilon$ ).

As quatro versões do Princípio da Incerteza/Indeterminação (duas ônticas e duas epistêmicas) estão resumidas abaixo na Tabela 1.

\section{Considerações Finais}

Cabe ressaltar que, embora percebamos que a distinção entre as diferentes categorias de um conceito pode ser considerada uma maneira simplista de apresentar a Física, tendo em vista que tais aspectos não são desenvolvidos de maneira isolada, identificamos, também, seu

\footnotetext{
11 Recentemente, Ozawa 65 66], desafiou a apresentação original de Heisenberg do Princípio da Incerteza, introduzindo "uma generalização da relação de Heisenberg comprovada como válida para medições arbitrárias é proposta e revela dois tipos distintos de possíveis violações da relação de Heisenberg". No entanto, Busch, Lahti \& Werner 67] mostraram que a relação de Ozawa não era universalmente válida.
} 
Tabela 1: Interpretações do Princípio da Incerteza.

\begin{tabular}{|c|c|c|}
\hline \multicolumn{3}{|c|}{ Interpretações do Princípio da Incerteza } \\
\hline & Versão & Principais Aspectos \\
\hline \multirow[t]{2}{*}{ Ônticas } & $\begin{array}{l}\text { Intrínseca } \\
(\Delta)\end{array}$ & $\begin{array}{l}\text { Refere-se a uma } \\
\text { indeterminação intrínseca na } \\
\text { estrutura da matéria. Quando } \\
\text { o momentum de uma } \\
\text { partícula é conhecido, suas } \\
\text { coordenadas não têm } \\
\text { significado físico. A discussão } \\
\text { de Heisenberg no início de } \\
1927 \text { sobre a descontinuidade } \\
\text { e a impossibilidade de } \\
\text { determinar a velocidade é um } \\
\text { exemplo dessa abordagem. }\end{array}$ \\
\hline & $\begin{array}{l}\text { Estatística } \\
(\sigma)\end{array}$ & $\begin{array}{l}\text { As indeterminações não se } \\
\text { referem a um único quantum, } \\
\text { mas a um conjunto. A } \\
\text { indeterminação deve ser } \\
\text { entendida como uma } \\
\text { divergência estatística. }\end{array}$ \\
\hline \multirow[t]{2}{*}{ Epistêmicas } & $\begin{array}{l}\text { Erro- } \\
\text { perturbação } \\
(\varepsilon-\eta)\end{array}$ & $\begin{array}{l}\text { No caso do microscópio de } \\
\text { raios gama, a posição é } \\
\text { realmente medida e } q_{1} \text { é o erro } \\
\text { de medição, } p \text {, entretanto, não } \\
\text { é medido e, portanto, } p_{1} \text { é a } \\
\text { perturbação máxima do } \\
\text { sistema. }\end{array}$ \\
\hline & $\begin{array}{l}\text { Erro-erro } \\
(\varepsilon-\varepsilon)\end{array}$ & $\begin{array}{l}\text { Refere-se à medição real da } \\
\text { posição (e seu erro) e } \\
\text { momentum (e seu erro). Nesse } \\
\text { caso, duas medições são } \\
\text { realizadas concretamente. }\end{array}$ \\
\hline
\end{tabular}

valor didático, pois, sabendo do nível de abstração do Princípio da Incerteza, entendemos que estudar cada aspecto isoladamente pode ajudar os estudantes a entender melhor o conceito.

Nesse contexto, tendo em vista o papel central do livro didático na formação de cientistas e professores de ciências, o presente trabalho pode ser compreendido como uma ferramenta de apoio para o Ensino de Física, uma vez que os livros didáticos não costumam distinguir entre as diferentes interpretações do Princípio da Incerteza e, geralmente, apresentam apenas algumas das possíveis formas de demonstração do mesmo. Sendo assim, como material complementar, o presente trabalho pode enriquecer o estudo do conceito e ajudar em sua compreensão.

Como pôde ser percebido, existem várias formas de demonstrar o Princípio da Incerteza e investir mais tempo na dedução, ao invés de fornecer os alunos as fórmulas prontas, pode contribuir para que os estudantes adquiram mais familiaridade com as equações e possam desenvolver seu raciocínio matemático. Além disso conexão do Princípio da Incerteza com sua fenomenologia pode tornar a compreensão, tendo em vista a aplicação do conceito, mais nítida, além de ressaltar a importância da experimentação, mesmo que mental, em uma teoria Física. Demonstrar a pluralidade interna do Princípio da Incerteza implica em demonstrar, também, a riqueza da
Teoria Quântica e contribui para passar aos estudantes uma noção de sua grande complexidade. Ademais, muito se discute sobre abordagens de Física Moderna na Ensino Médio, mas só podemos falar em ensinar Física Quântica na educação básica se discutirmos, primeiro, a formação de professores.

Uma possível maneira de trabalhar o presente material é através de uma abordagem didática que, complementar ao estudo do livro, permita dividir os alunos segundo qual das três diferentes categorias mais se identifiquem. Tal abordagem, apesar de possuir suas limitações, respeitaria a pluralidade existente em sala de aula, a diversidade dos alunos, e permitiria, assim, atingir mais estudantes e em níveis de abstração mais profundos.

\section{Material Suplementar}

O seguinte material suplementar está disponível online: Apêndice A: Postulados da Mecânica Quântica Apêndice B: Conceitos da Teoria Estatística Apêndice C: Desigualdade de Cauchy-Schwarz

\section{Referências}

[1] W. Heisenberg, em Quantum Theory and Measurement, editado por J.A. Wheeler e W.H. Zurek (Princeton University Press, Princeton, 1983).

[2] M. Jammer, The Conceptual Development of Quantum Mechanics (McGraw-Hill Book Company, New York, 1966).

[3] W. Pauli, General Principles of Quantum Mechanics (Springer, New York, 1980).

[4] A. Einstein, B. Podolsky e N. Rosen, Phys. Rev. 47, 777 (1935).

[5] D. Bohm, Phys. Rev. 85, 166 (1952).

[6] M.R. Bento, N.W. Lima e C.J.H. Cavalcanti, em: XVIII Encontro Pesquisa em Ensino Física (Florianópolis, 2020).

[7] R.̈̈. Akyüz, Am. J. Phys. 56, 997 (1988).

[8] E. Arthurs e M.S. Goodman, Phys. Rev. Lett. 60, 2447 (1988).

[9] D.N. Williams, Am. J. Phys. 47, 606 (1979).

[10] G.W. Forbes e M.A. Alonso, Am. J. Phys. 69, 1091 (2001).

[11] V. Gattus e S. Karamitsos, Eur. J. Phys. 41, 065407 (2020).

[12] L. Goldenberg e L. Vaidman, Am. J. Phys. 64, 1059 (1996).

[13] J. Lévy-Leblond, Am. J. Phys. 54, 135 (1986).

[14] J.H. Marburger III, Am. J. Phys. 76, 585 (2008).

[15] M.C. Palenik, Eur. J. Phys. 35, 045014 (2014).

[16] J. Peslak Jr, Am. J. Phys. 47, 39 (1979).

[17] A. Tamura e Y. Tatsumi, Eur. J. Phys. 38, 065402 (2017).

[18] S.P. Boughn e M. Reginatto, Eur. J. Phys. 39, 035402 (2018).

[19] H.C. Corben, Am. J. Phys. 47, 1036 (1979).

[20] B.G. Williams, Am. J. Phys. 52, 425 (1984). 
[21] P. Hadzidaki, Sci. Educ. 17, 613 (2008).

[22] J. Hilgevoord e J.B.M. Uffink, Eur. J. Phys. 6, 165 (1985).

[23] V. Jijnasu, Studies in History and Philosophy of Modern Physics 55, 62, (2016).

[24] M.G. Raymer, Am. J. Phys. 62, 986 (1994).

[25] P.J. Riggs, Eur. J. Phys. 39, 035409 (2018).

[26] J. Maziero, Rev. Bras. Ensino Física 39, e4306 (2017).

[27] S.S. Chibeni, Rev. Bras. Ensino Física 27, 181 (2005).

[28] C. Singh, Am. J. Phys. 69, 885 (2001).

[29] R. Karam, Am. J. Phys. 86, 245 (2018).

[30] R. Karam, Am. J. Phys. 88, 39 (2020).

[31] N. Lima e R. Karam, Am. J. Phys. 89, 521 (2021).

[32] N. Lima, C. Cavalcanti e F. Ostermann, Rev. Bras. Ensino Física 43, e20200270 (2021).

[33] M. Jammer, The Philosophy of Quantum Mechanics (John Wiley and Sons, New York, 1974).

[34] O. Pessoa Jr., Conceitos de Física Quântica (Livraria da Física, São Paulo, 2005), v.1.

[35] Y.W. Cheong e J. Song, Sci \& Educ. 23, 1011 (2014).

[36] W. Heisenberg, Zeitschrift für Phys. 33, 879 (1925).

[37] M. Born, The statistical interpretation of quantum mechanics - Nobel Lecture, 1951, disponível em: https: //www.nobelprize.org/prizes/physics/1954/born/le cture/, acessado em 01/03/2021.

[38] M. Born e P. Jordan, Zeitschrift für Phys. 34, 858 (1925).

[39] M. Born, W. Heisenberg e P. Jordan, Zeitschrift für Phys. 35, 557 (1926).

[40] W. Pauli Jr, Zeitschrift für Phys. a Hadron. Nucl. 36, 336 (1926).

[41] P.A.M. Dirac, Proc. R. Soc. Lond. A 109, 642 (1925).

[42] P.A.M. Dirac, Proc. R. Soc. Lond. A 112, 661 (1926).

[43] P.A.M. Dirac, Proc. R. Soc. Lond. A 113, 621 (1927).

[44] P. Jordan, Zeitschrift für Phys. 40, 809 (1927).

[45] P. Jordan, Zeitschrift für Phys. 44, 1 (1927).

[46] E. Schrodinger, Collected Papers on Wave Mechanics (Backie \& Son Limited, London, 1928).

[47] A. Einstein, em: The Collected Papers of Albert Einstein, Volume 14: The Berlin Years: Writings \& Correspondence, April 1923-May 1925, editado por D.K. Buchwald, J. Illy, Z. Rosenkranz, T. Sauer e O. Moses (Princeton University Press, Princeton, 2015), p. 418.

[48] M. Beller, Quantum dialogue: the making of a revolution (The University of Chicago Press, Chicago, 1999).

[49] W. Heisenberg, A parte e o todo (Contraponto, Rio de Janeiro, 1996).

[50] W. Heisenberg, Physics and Philosophy (Penguin Books, London, 2000).

[51] C. Cohen-Tannoudji, B. Diu e F. Laloë, Quantum Mechanics (Wiley Interscience, New York, 1991).

[52] D.J. Griffiths, Introduction to Quantum Mechanics (Pearson Prentice Hall, New Jersey, 2005).

[53] H. Weyl, The Theory of Groups \& Quantum Mechanics. (Dover Publications, New York, 1950).

[54] H.P. Robertson, Phys. Rev. 34, 163 (1929).

[55] E. Schrodinger, arXiv:quant-ph/9903100v2 (2014).

[56] L.D. Landau e E.M. Lifshitz, Quantum Mechanics: nonrelativistic theory (Pergamon Press, Oxford, 1991).

[57] V.V. Dodonov, Phys. Rev. A 97, 022105 (2018).
[58] J.E.G. Farina, Int. J. Theor. Phys. 21, 83 (1982).

[59] J.A. da Cruz e N.W. Lima, em: I Encontro sobre História e Filosofia no ensino de Física do sul do Brasil (Passo Fundo, 2021).

[60] D. Halliday e R. Resnick, Fundamentos de Física: Óptica e Física Moderna (LTC, Rio de Janeiro, 2009).

[61] P. Busch, T. Heinonen e P. Lahti, Phys. Rep. 452, 155 (2007).

[62] P. Busch e G. Jaeger, Found. Phys. 40, 1341 (2010).

[63] K. Popper, The Logic of Scientific Discovery (Routledge, New York, 2002).

[64] M. Bunge, Física e Filosofia (Perspectiva, São Paulo, 2015).

[65] M. Ozawa, Phys. Lett. A. 318, 21 (2003).

[66] M. Ozawa, Phys. Rev. A, 67, 042105 (2003).

[67] P. Busch, P. Lahti e R.F. Werner, Rev. Mod. Phys. 86, 1261 (2014). 